respiratory complications: Prospective propensity score matched cohort study. BMJ 2012; 345:e6329

5. Meyer MJ, Bateman BT, Kurth T, Eikermann M: Neostigmine reversal doesn't improve postoperative respiratory safety. BMJ 2013; 346:f1460

6. Naguib M, Kopman AF, Lien CA, Hunter JM, Lopez A, Brull SJ: A survey of current management of neuromuscular block in the United States and Europe. Anesth Analg 2010; 111:110-9

7. Kopman AF, Eikermann M: Antagonism of non-depolarising neuromuscular block: Current practice. Anaesthesia 2009; 64(suppl 1):22-30

(Accepted for publication February 25, 2015.)

\section{Confounders versus Mediators: An Important Distinction}

\section{To the Editor:}

We thank Kashy et al. ${ }^{1}$ for their interesting analysis ("Effect of Hydroxyethyl Starch on Postoperative Kidney Function in Patients Having Noncardiac Surgery)." However, we disagree with some of their methods/assumptions and, in fact, reached different conclusions with the same data. As shown in table $1,{ }^{1}$ the authors did not match patients on intraoperative characteristics. Accordingly, the two groups being compared (intraoperative hydroxyethyl starch [HES] recipients $v$ s. noncolloid recipients) were significantly different. After propensity matching, nearly twice as many HES recipients were hypotensive (37 vs. 20\%), nearly three times as many received blood transfusions intraoperatively (14 vs. $5 \%$ ), and nearly one-and-a-half times as many were likely to have received vasopressors (70 vs. 45\%). In addition, blood loss was twice as much as among noncolloid recipients than among HES recipients (on average 200 vs. $100 \mathrm{ml}$ ). Hence, these groups are not comparable "at baseline."

As shown in figure $2,{ }^{1}$ blood loss and hypotension are correctly considered confounders (i.e., may be associated with both predictor and outcome) and are controlled for in analysis. In contrast, the authors state that intraoperative vasopressor use and intraoperative blood product transfusion might be mediators (i.e., "mechanisms by which HES administration might cause increased risk of AKI [acute kidney injury]"), implying a position in the causal pathway. Are the authors claiming that AKI (occurring as a result of intraoperative exposure to HES) might occur via HES leading to intraoperative vasopressor use and/or blood product transfusion? HES has been shown to influence hemostasis adversely. ${ }^{2}$ Are the authors saying that HES-associated AKI may be a result of HES-induced coagulopathy (leading to increased blood product transfusion)?

We believe that intraoperative HES therapy (among patients undergoing major noncardiac surgery) is probably related to clinician-perceived hypovolemia (absolute or relative). Such hypovolemia (rather than receipt of HES per se) may lead to both vasopressor use (secondary to hypotension), transfusion, and to AKI, that is, residual confounding is very likely. This is apparent in the sensitivity analysis (table 3), ${ }^{1}$ as the model with transfusion and vasopressor use as potential confounders showed no effect of HES on AKI (odds ratio, $1.10 ; 95 \% \mathrm{CI}, 0.96$ to $1.25 ; P=0.12$ ).

The authors might also consider an instrumental variable approach (with calendar time as the instrument). The discontinuation of intraoperative HES use is essentially a "pseudorandom event" such that patients presenting for noncardiac surgery before the HES withdrawal date are probably very similar to patients presenting after this date (of course, all relevant baseline characteristics need to be tabulated to ensure that comparability exists, and where it does not, the parameter that is dissimilar between groups needs to be controlled for if it is a confounder). Such an observational study would emulate the "ideal" randomized controlled trial where essentially similar patients receive different interventions.

\section{Competing Interests}

The authors declare no competing interests.

Karthik Raghunathan, M.D., M.P.H., Timothy E. Miller, M.B., Ch.B., F.R.C.A., Ali M. Rashid, M.D. Durham VA Medical Center, Duke University Medical Center, Durham, North Carolina (K.R.).kr118@duke.edu

\section{References}

1. Kashy BK, Podolyak A, Makarova N, Dalton JE, Sessler DI, Kurz A: Effect of hydroxyethyl starch on postoperative kidney function in patients having noncardiac surgery. ANESTHESIOLOGY 2014; 121:730-9

2. Kozek-Langenecker SA: Effects of hydroxyethyl starch solutions on hemostasis. ANesthesiology 2005; 103:654-60

(Accepted for publication February 25, 2015.)

\section{High-molecular Hydroxyethyl Starch: Is More Data Still Needed?}

\section{To the Editor:}

With some degree of amazement, we read the article by Kashy et al. ${ }^{1}$ on the influence of $6 \%$ hydroxyethyl starch (HES) 670/0.75 (Hextend; Hospira Inc., USA) on perioperative acute kidney injury in patients undergoing noncardiac surgery. The data are derived from a database of more than 120,000 patients treated in Cleveland hospitals, in which 6\% HES 670/0.75 was the most commonly used colloid between 2005 and 2012. After propensity-matched multivariable analysis, the authors found a higher risk of developing more severe acute kidney injury with the use of 6\% HES 670/0.75 as compared with sole crystalloids. Notably, a higher rate of acute kidney injury had already been shown with the use of high-molecular HES (6\% HES 200/0.62) in critically ill patients with sepsis as compared with gelatin in 2001. ${ }^{2}$ Moreover, direct comparison of lowmolecular (6\% HES 130/0.4) versus high-molecular HES 